\title{
Antisense to glucosylceramide synthase in human neuroepithelioma affects cell growth but not apoptosis
}

\author{
DOI: $10.1038 / \mathrm{sj} / \mathrm{cdd} / 4401040$
}

\section{Dear Editor,}

Ceramide (Cer) glucosylation provides the key metabolic step whereby cells initiate the synthesis of a wide range of highorder neutral glycosphingolipids (GSLs) and gangliosides. Glucosylceramide synthase (GCS), the enzyme catalyzing the transfer of glucose from UDP-glucose to Cer, is a transmembrane protein localized in the cis/medial Golgi, with a N-terminal signal-anchor sequence and a C-terminal catalytic domain located in the cytoplasm. ${ }^{1}$ Once synthesized, glucosylceramide (GlcCer) may be flipped to the inner layer of the Golgi membranes and further processed to higher-order GSLs by the sequential addition of sugar residues. ${ }^{1}$ Owing to its key position in this biosynthetic pathway and considering the wide range of biological processes in which GSLs have been involved, GCS activity is expected to markedly modulate cell functions. ${ }^{2-6}$ Moreover, the importance of GCS in tissue homeostasis and development has been demonstrated by the targeted disruption of the gene which results in gross embryonic defects and early death of the embryo. ${ }^{7}$ An additional role for GCS is given by the fact that Cer and GlcCer, the substrate and the reaction product of the enzyme, are themselves active molecules, allowing the triggering of alternative cell responses. Cer, in particular, can be generated by neosynthesis or sphingomyelin hydrolysis after cell exposure to a variety of stimuli and has been implicated in induction of growth arrest and/or apoptosis. ${ }^{8}$ Glucosylation provides a major pathway for Cer downregulation and the quenching of its effects: thus, it has been proposed that GCS inhibition may induce growth arrest via Cer accumulation ${ }^{9}$ and that the multidrug-resistance state of cancer cells may be associated with elevated GCS activity and reversable by inhibition of Cer glucosylation. ${ }^{10-12}$ Indeed, it has been reported that synthetic GCS inhibitors may affect cell growth and morphology; ${ }^{13}$ moreover, these compounds have been reported to sensitize tumor cells to the cytotoxic action of various anticancer compounds. ${ }^{14,15}$ Nevertheless, evidence has been recently provided for unspecific actions of these inhibitors. For instance, DL-threo-1-phenyl-2-decanoylamino3-morpholino-1-propanol (PDMP) has been reported to affect calcium homeostasis and membrane flow, ${ }^{16}$ as well as thymidine transport, ${ }^{17}$ independently from its effects on glycosphingolipid metabolism. These observations suggest that data obtained using these inhibitors must be interpreted with caution. In order to study the biological role of GCS by an alternative approach, we have transfected CHP-100 human neuroepithelioma cells with a GCS antisense vector (GCSas). Stable transfection of CHP-100 cells with GCSas by lipofection resulted in the generation of fifteen G418 resistant clones. These were isolated and selected by measuring GCS activity using the synthetic fluorescent substrate $\mathrm{C}_{6}-\mathrm{NBD}$. The screening resulted in various clones which exhibited a stable decrease in GCS specific activity of about $60 \%$ (in this report we have used the CHP-AS6 and CHP-AS13) in comparison to vector control cells the CHP-Neo $\left({ }^{*} P<0.01\right)$ (Figure $\left.1 \mathrm{~A}\right)$. It is important to note that there was no difference in GCS activity between CHP-Neo and parental CHP-100 cells (Figure 1A). Transfected cells were further characterized by analyzing the expression of GCS antisense RNA (Figure 1B) and GCS protein levels (data not shown). A representative RT-PCR electophoresis gel showed that the selected clones expressed high levels of antisense GCS RNA (Figure 1B).

Downregulation of GCS activity resulted in marked effects on the sphingolipid pattern, with Cer levels being elevated and GSLs expression diminished. Figure 1C shows that both GCSas selected clones displayed increased Cer levels in comparison to CHP-Neo: in particular, Cer concentration was increased by fivefold in CHP-AS6 and by threefold in CHP-AS13. Figure 1D shows that levels of neutral GSLs were not significantly diminished in the two GCSas clones; on the other hand, GCS downregulation markedly affected the ganglioside pattern. However, GSLs expression was not broadly and uniformly reduced; rather, we found that the residual GlcCer synthesized is utilized to maintain fairly constant levels of neutral GSLs and GM3, to the detriment of the expression of higher order gangliosides. The glycosyltransferases operating the sequential addition of carbohydrate residues to GlcCer are not uniformly distributed in the Golgi apparatus: thus, whereas globotriaosylceramide and GM3 are synthesized in the cis-Golgi, synthesis of high order gangliosides predominantly occurs in the trans compartment. ${ }^{18,19}$ This leaves open the possibility that differences in ganglioside composition, as observed in GCSas clones in comparison to CHP-Neo, may also be due to impaired traffic between the two Golgi compartments.

In various reports the upregulation of GCS has been related to the resistance displayed by tumor cells to anticancer drugs inducing apoptosis through Cer elevation. In particular, studies performed on MCF-7 breast cancer cells have shown that resistance to doxorubicin can be conferred by GCS overexpression and reversed by GCS downregulation by the antisense strategy. ${ }^{12,20}$

As mentioned above, both GCSas selected clones displayed increased Cer levels in comparison to CHP-Neo (Figure 1C); nevertheless, as shown in Figure $1 \mathrm{E}$ no substantial difference in basal apoptosis was observed between GCSas clones and CHP-Neo cells. The reasons 


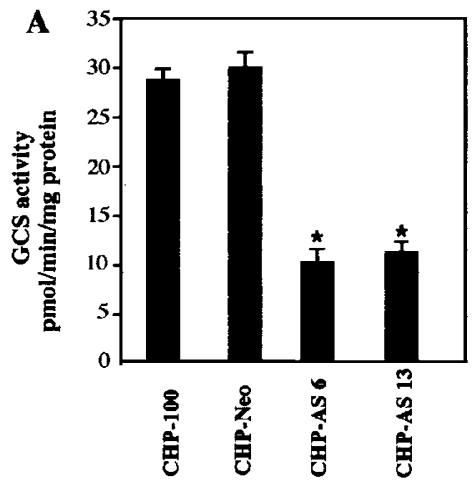

$\mathbf{E}$
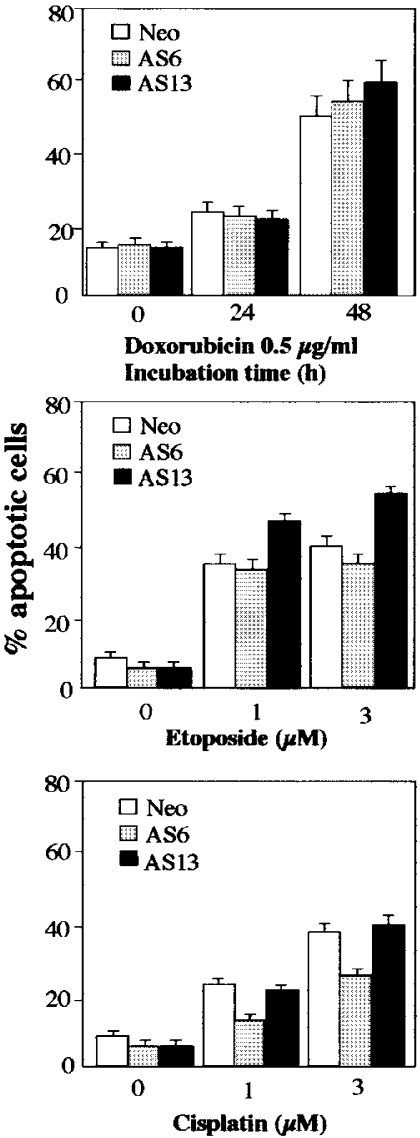

B

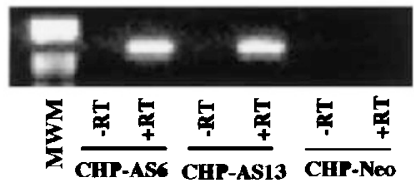

C Ceramide (pmol/nmol lipid phosphate)

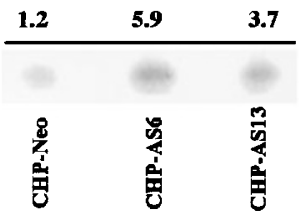

D

\begin{tabular}{|c|c|c|c|}
\hline & CHP-Neo & CHP-AS6 & CHP-AS13 \\
\hline Neutral GSLs & \multicolumn{3}{|c|}{$\mu \mathrm{g}$ glycosphingolipid/mg protein } \\
\hline GlcCer & $0.16 \pm 0.03$ & $0.12 \pm 0.02$ & $0.18 \pm 0.03$ \\
\hline Lact-Cer & $0.08 \pm 0.03$ & $0.06 \pm 0.01$ & $0.08 \pm 0.02$ \\
\hline $\begin{array}{l}\text { GbOse3-Cer } \\
\text { Gangliosides }\end{array}$ & $0.18 \pm 0.01$ & $0.16 \pm 0.03$ & $0.21 \pm 0.04$ \\
\hline GM3 & $1.14 \pm 0.14$ & $0.90 \pm 0.12$ & $1.13 \pm 0.14$ \\
\hline GM2 & $3.57 \pm 0.43$ & $2.19 \pm 0.30\left(^{*}\right)$ & $2.22 \pm 0.32$ \\
\hline GM1 & $2.39 \pm 0.32$ & $0.79 \pm 0.10(*)$ & $1.39 \pm 0.16$ \\
\hline GDla & $1.39 \pm 0.17$ & $0.23 \pm 0.04(*)$ & $0.47 \pm 0.07$ \\
\hline
\end{tabular}

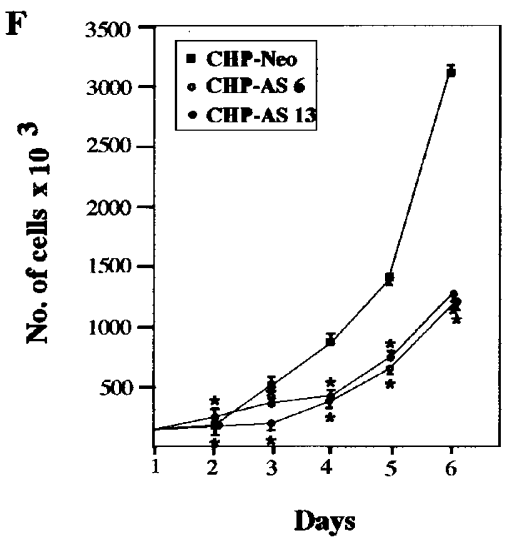

Figure 1 (A) GCS activity was measured in CHP-100 cells, CHP-Neo vector control cells and GCSas transfected cells using the synthetic fluorescent substrate $\mathrm{C}_{6}$-NBD. (B) GCS antisense mRNA expression. Total RNA isolated from CHP-AS6, CHP-A13 and CHP-Neo control cells, was reverse trascribed and amplified by RT - PCR with GCS antisense specific primers. (C) HPTLC analysis of endogenous Cer levels in CHP-Neo and GCSas clones. Lipids extracts from CHP-Neo, CHPAS 6 and CHP-A13 clones (1.5 mg protein each sample) were resolved by HPTLC. Lipid spots were revealed by cupric acetate/phosphoric acid charring and Cer identified by comigration with authentic standards. Plates were scanned with a Umax model scanning densitometer, Cer band intensities were calculated and referred to those of known amounts of standards for quantitation. Data are representative of three other experiments. (D) Neutral glycosphingolipids and gangliosides were resolved by HPTLC and visualized by $\alpha$-naphthol/sulphuric acid or resorcinol hydrochloride reagents, respectively. Plates were scanned with a Umax model scanning densitometer. The relative band intensities were calculated and referred to those of known amounts of standards for quantitation. Results are means \pm S.D. of three independent determinations. Statistical significance: ${ }^{*} P<0.01$, as compared to untreated samples, as from Student's $t$-test. (E) CHP-Neo, CHP-AS6 and CHP-AS13 clones were treated with $0.5 \mu \mathrm{g} / \mathrm{ml}$ doxorubicin for the indicated times; 1 or $3 \mu \mathrm{M}$ etoposide for $48 \mathrm{~h}$ and 1 or $3 \mu \mathrm{M}$ cisplatin for $48 \mathrm{~h}$ Apoptosis was monitored by evaluation of the pre- $G_{1}$ cell population, after flow cytometric analysis. Results are means \pm S.D. of three indipendent determinations. The apparent increase in apoptosis with the AS13 clone with 1 and $3 \mu \mathrm{M}$ etoposide and the apparent decrease with clone AS6 with 1 and $3 \mu \mathrm{M}$ cisplatin is not significant. (F) $3 \times 10^{4} \mathrm{CHP}$-Neo cells and GCSas clones were seeded into 60 -mm dishes and cultured for up to 6 days. Each day cells from each dish were removed by trypsinization and counted by Trypan blue exclusion. Results are means \pm S.D. of three independent determinations. Statistical significance: ${ }^{*} P<0.01$, as compared to control cells, as from Student's $t$-test 
of this apparent discrepancy remain to be elucidated: nevertheless it must be mentioned that endogenous ceramide elevation does not necessarily perform an apoptotic action; moreover, several concerns have been recently cast about the possibility that short-chain and endogenous Cer share the same biological properties. ${ }^{21,22}$ In addition, we studied whether downregulation of GCS expression potentiated the apoptotic response of CHP-100 cells to various cytotoxic agents. In keeping with our previous results showing that the apoptotic action of doxorubicin in CHP-100 cells is not mediated by Cer, ${ }^{23}$ we found that drug induced apoptosis in GCSas clones was not increased in comparison to CHP-Neo cells (Figure 1E). A similar result, however, was also observed for etoposide and cisplatin, namely two other anticancer drugs, reported to induce apoptosis through intracellular Cer elevation in other cell lines ${ }^{24,25}$ (Figure 1E). These results suggest that endogenous Cer elevation does not invariably perform an apoptotic action. It remains to be studied whether, as observed for doxorubicin, the apoptotic action of etoposide and cisplatin in CHP-100 cells is dissociated from Cer generation. Nevertheless our study shows that the level of GCS expression is not a general determinant of cell sensitivity to anticancer drug-treatment.

Conversely GCSas clones displayed a decrease in cellcell adhesion, which resulted in an evident cell scattering, and a reduced cell-substratum adhesion although no detectable difference in the expression of molecules controlling cell-cell (such as E-cadherin or $\alpha$ and $\beta$ cathenins) as well as cell-substratum interaction (such as $\alpha 1, \alpha 3$ and $\alpha 5 \beta 1$ integrins) was observed in any cell type (data not shown). However, the GCSas clones exhibited a significant decline of the growth rate, as compared to that of CHP-Neo cells (Figure 1F). This marked difference in cell growth appears to be a specific effect elicited by GCS inhibition: in fact, CHP-Neo cells did not show any detectable difference in growth when compared to wildtype CHP-100 cells. Both PDMP and the more potent GCS inhibitor DL-threo-1-phenyl-2-hexadecanoylamino-3-pyrrolidino-1-propanol have been demonstrated to reduce tumor growth and metastatization in vivo. ${ }^{26}$ It has been predicted that impairment of GCS activity affects the cell phenotype by downregulating GSL levels ${ }^{13}$ a notion well supported by the evidence that GSLs may provide the site of attachment for various receptors mediating cell to cell adhesion ${ }^{4-6}$ and are involved in the modulation of integrin function. ${ }^{27}$ Moreover, there is evidence that GSLs are clustered in discrete areas of the plasma membrane, referred to as 'glycosphingolipid signaling domains', with proteins involved in cell adhesion and signal transduction. ${ }^{28}$ It is possible to hypothesize that, in addition to the higher Cer content, ganglioside depletion could contribute to the phenotype displayed by the selected GCSas clones.

\section{Acknowledgements}

The authors would like to express their gratitude to Professor F Autuori for stimulating discussions. The work was partially supported by grants from: European Community Apoptosis Mechanisms', AIRC and AIDS' project from Ministero Sanita' to M Piacentini. F Di Sano was partially supported by a fellowship by FIRC.

\author{
F Di Sano ${ }^{1}$, S Di Bartolomeo ${ }^{1}$, B Fazi ${ }^{1}$, C Fiorentini?, \\ $P$ Matarrese $^{2}$, A Spinedi $^{1}$ and M Piacentini ${ }^{*, 1,3}$ \\ 1 Department of Biology , University of Rome Tor Vergata', Rome, Italy; \\ 2 Laboratorio Ultrastrutture, Istituto Superiore di Sanità, Italy; \\ ${ }^{3}$ Cell Biology and E.M. Unit IRCCS Lazzaro Spallanzani', Rome, Italy \\ * Corresponding author: M Piacentini, Cell Biology and EM Unit IRCCS \\ 'Lazzaro Spallanzani', Rome, Italy. Tel: 39067259 4370; \\ Fax: 39062023 500; E-mail: mauro.piacentini@uniroma2.it
}

\footnotetext{
1. Ichikawa S and Hirabayashi Y (1998) Trends Cell Biol. 8: 198-202

2. Bremer EG et al. (1986) J. Biol. Chem. 261: 2434-2440

3. Mutoh T et al. (1998) J. Biol. Chem. 273: 26001-26007

4. Handa K et al. (1991) Biochem. Biophys. Res. Commun. 181: 1223-1230

5. Handa K et al. (1997). Biochemistry. 36: 12412-12420

6. Kopitz J et al. (1998) J. Biol. Chem. 273: 11205-11211

7. Yamashita T et al. (1999) Proc. Natl. Acad. Sci. USA. 96: 9142-9147

8. Hannun YA and Luberto C (2000) Trends Cell Biol. 10: 73-80

9. Rani CS et al. (1995) J. Biol. Chem. 270: 2859-2867

10. Lavie $Y$ et al. (1997) J. Biol. Chem. 272: 1682-1687

11. Lucci A et al. (1998) Anticancer Res. 18(1B): $475-480$

12. Liu YY et al. (2000) J. Biol. Chem. 275: 138-143

13. Barbour S et al. (1992) J. Cell Physiol. 150: 610-619

14. Sietsma H et al. (2000) Clin. Cancer Res. 6: 942-948

15. Olshefski RS and Ladisch S (2001) Int. J. Cancer 93: 131-138

16. Kok JW et al. (1998) J. Cell Biol. 142: 25-38

17. Griner RD and Bollag WB (2000) J. Pharmacol. Exp. Ther. 294: 1219-1224

18. van Echten $\mathrm{G}$ et al. (1990) Eur. J. Cell Biol. 51: 135-139

19. Iber H et al. (1992) J. Neurochem. 58: 1533-153723

20. Liu YY et al. (1999) J. Biol. Chem. 274: 1140-1146

21. Hofmann K and Dixit VM (1998) Trends Biochem. Sci. 23: 374-377

22. Di Bartolomeo S and Spinedi A (2001) Biochem. Pharmacol. 61: 851-856

23. Di Bartolomeo S et al. (2000) J. Neurochem. 75: 532-539

24. Sawada M et al. (2000) Cell Death Differ. 7: 761-772

25. Noda S et al. (2001) J Neurooncol. 52: 11-21

26. Deng W et al. (2000) J. Natl. Cancer Inst. 92: 912-917

27. Zheng M et al. (1993) J. Biol. Chem. 268: 2217-2222

28. Iwabuchi K et al. (1998) J. Biol. Chem. 273: 9130-9138
} 